\title{
LEAST PROTECTIVE PART OF THE BODY DURING DENTAL CARE --YOUR EYES
}

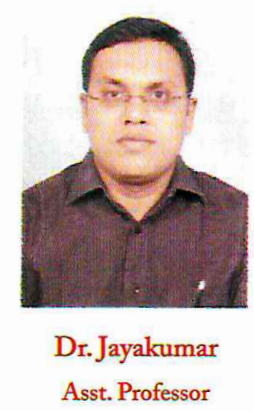

\author{
Dept. Of Conservative Dentistry \& \\ Endodontics
}

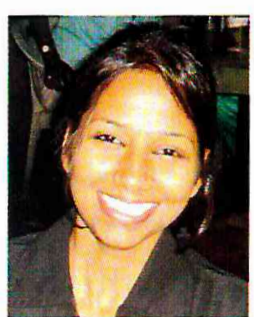

Dr. Sonia

P.G. Student

Abstract: Protective eyewear is integral component of Personnel protective equipments (PPE) and helps contribute to a safer work environment. Protective eyewear protocol is important in reducing contamination of the mucous membranes and skin surrounding the eyes, nose as well as injury and infections. The topic of occupational eye injuries in dentistry is one that clearly requires further study, thus, this article reviews the various eye injuries and their prevention in dentistry.

Both dental personnel and patients are at risk of suffering eye-related injuries during dental procedures. Six categories of common ocular injuries in the oral care environment are as:

1) Physical trauma

2) Microbial infection

3) Chemical

4) Fatigue

5) Visible light curing unit

6) Lasers/electromagnetic radiation

Physical eye trauma: High-speed and ultra high speed handpieces rotate at 180,000 to 500,000 rpm causing particles to be projected up to $50 \mathrm{mph}$. Projectiles include pieces of amalgam, tooth enamel, calculus, pumice, and broken dental burs. In most cases the foreign body locates itself in the conjunctival sac or the cornea, causing acute pain, lacrimation and a reddening of the eyeball and more serious perforation of the cornea and a consequent injury to the lens.

Microbial infection: Bacteria-laden calculus, coolant spray or mist associated with high-speed handpieces and ultrasonic scalers can transmit bacteria, which may contaminate the unprotected eyes and the respiratory system. Mycobacterium tuberculosis, staphylococci, streptococci, herpes simplex virus (Herpetic Keratitis - the second leading cause of blindness), cytomegalovirus, and hepatitis $\mathrm{B}$ and $\mathrm{C}$ are examples of pathogenic microorganisms transmitted in the dental practice setting. Ocular herpes is a risk even without signs of intraoral or extraoral herpetic lesions because the virus is often present in the saliva before clinical symptoms.

Chemical Trauma: Substances such as varnish, bleach, formocreosol, eugenol, hydrogen peroxide, sodium hypochlorite, phosphoric acid, methyl methacrylate, chloroform, glutaraldehyde, alcohol, trichloracetic, chromic acids and $\mathrm{x}$-ray photochemicals and apart from this, Plaster of paris which contains small quantities of lime and quartz, can damage the eye; whilst pumice can abrade it.

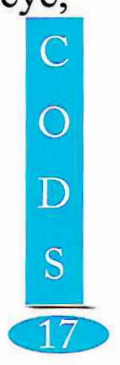


Fatigue: Prolonged visual strain leads to overall exhaustion of the organism. Among its many symptoms the foremost is eye fatigue. It reveals itself as the sensation of heavy eyelids, burning and stinging under the eyelids and deteriorated vision. This is usually accompanied by bloodshot eyes, blinking, lacrimation and increased sensitivity of the eyeball to touch. Very often headaches and eye pain join in. Eye fatigue causes a decrease in critical fusion frequency, a delayed accommodative reflex, a reduced accommodation width and a shift of refraction towards myopia or hypermetropia. It is impossible to distinguish between symptoms of general fatigue and fatigue of the eye.

Visible light curing unit: Evidence exists that photochemical injury to the retina can occur as a result of exposure to the short-wavelength light. The toxic effect consists in hypersensitivity to light, which manifests itself in a burning sensation, a reddening of the eye or the appearance of urticaria. As a result of a photoimmunological reaction eczema might occur.

\section{Guidelines for the Use of Visible Light Cure Units}

1. Never look directly at the light and do not point it toward others.

2. Do not look directly at scattered light or tooth glow for more than one second.

3. Use the delay switch so the tip can be placed properly before the light is activated

4. If the unit comes with a filter for the tip, use it as directed by the manufacturer.

5. Shield the operating team from the light by using your thumb or a mirror as a barrier.

6. When possible, distribute the operation of VLC units evenly between dental team members.

7. Use filtered spectacle lenses if you are doing a high volume of procedures requiring light-cured materials.

8. Choose a tinted lens that effectively blocks light in the range below $500 \mathrm{~nm}$.

9. The light of such units should be avoided by individuals sensitive to light or treated for the same, or those who take photosensitizing drugs.

Lasers induced eye hazards: Laser damage to the eye can lead to headaches, excessive watering of the eyes and floaters (shortly after the exposure). Damage to the cornea will be uncomfortable, even painful, and cause a gritty sensation, like sand in the eye. Cataracts can result from lasers in the 1,4003,000 nm range (such as the dental lasers Er: Cr: YSGG and Er: YAG). A $10,600 \mathrm{~nm} \mathrm{CO}$ laser can cause a burning pain on the cornea if exposure occurs and a severe burn to the cornea may result in permanent scarring and partial loss of vision. Retinal damage may go unnoticed and can result in blindness, an inability to distinguish color, or diminished reading, working, and/or night vision. Sometimes an audible pop can be heard at the time of the exposure. The energy of a laser can be intensified 100,000 times on the retina due to the focusing effects of the lens and cornea.

\section{Protective Eyewear}

As required by OSHA, dental staff should wear a either a face shield or shatter-resistant eyeglasses with side shields when performing or assisting any procedure that could result in projectiles, chemicals, or aerosols entering the eye.

Three basic types of protective eyewear are available: glasses, goggles, and face shields. Ideal characteristics of each type of eyewear include: impact resistance, light weight, scratch resistant, distortion free and comfortable for the practitioner. The closer the eyewear fits to the face, the better the quality of the seal provided.

A common mistake made by oral health care practitioners who wear prescription eyeglasses is wearing them as safety eyewear. Contemporary prescription glasses often consist of small frames with small lens diameters, which are often not impact resistant, and provide minimal eye protection from flying debris and chemicals. 
Wraparound glasses or glasses with solid side shields prevent microorganisms and debris from entering the sides of the eyewear. Disposable plastic add-on side shields are available for clinicians with prescription eyewear although many add-on shields do not adapt well to the sides of the face and minimize their effectiveness (Figure 1). If add-on side shields are used, they need to be solid and fit tightly against the sides of the glasses and the face. For prescription eyeglass wearers, ANSI-certified goggles or full face shields can be used over prescription eyewear. This combination, however, may prove cumbersome and uncomfortable for the clinician (Figure 2). Another option is to have the lens prescription placed into impact resistant safety glasses (Figure 3 ). During procedures where a large amount of splatter is produced, a clear face shield may be useful.

For maximum protection and comfort a combination of features is available among eyewear manufacturers that contribute to their wearability. In addition to solid impact resistant side shields, adjustable side arms, brow guards, anti-fog features, and mask hooks are available.

For treatment with lasers protective eyewear must be specific to the laser wavelength that is being used and must have side shields. The filter that coats the goggles and inserts can decrease visual acuity during laser procedures. The temptation to lift the protective eyewear during laser operation to observe the tissue interaction more closely must be avoided and performed only when the laser is in the safe "stand-by" mode.
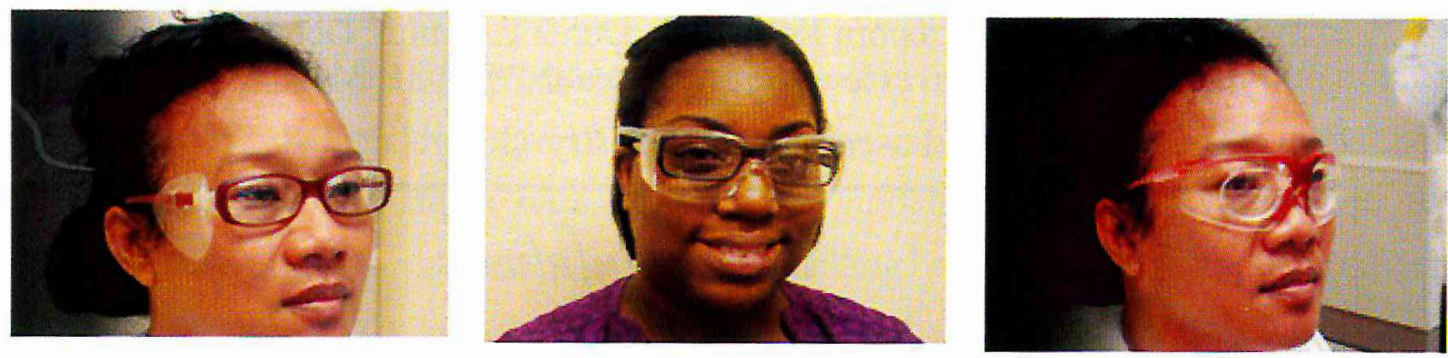

\section{Patient Protection}

Equally important is patient eye protection, which is a vital safeguard against injury, infection, and potential lawsuits. With patients' eyes less than $5 \mathrm{~cm}$ from the work area, left unprotected, the possibility of injury and infection is high. With the advent of four-handed dentistry, the patient's risk for eye trauma has increased. Dental items should be transferred over the patient's chest rather than over the face. Patients who wear glasses should continue to wear them during treatment. Safety goggles should be provided for patients who do not normally wear glasses.

\section{Care of Eyewear}

Since many chemical disinfectants can cause eyewear distortion over time as well as irritation to the eyes, they should be used with caution. The protective film on goggles and inserts used with lasers will degenerate over time; improper storage, cleaning, and handling will accelerate that degeneration. Surface disinfectant sprays are too harsh and can scratch the protective coating. The Standard Proficiency Certification course from the ALD recommends washing protective eyewear with antibacterial soap and drying the eyewear with a soft cotton cloth. Alcohol-based sanitizers especially developed for eyewear are a good choice for disinfection. Inserts and goggles must be inspected frequently for any cracks, peeling,

blisters, cloudiness, occlusions, frostiness, discolorations, crazing, and scratches that could reduce or eliminate the protection these goggles provide. 


\section{Conclusion}

Dental face masks and eyewear are not created equal nor do all dental procedures involve equal risks of exposure. Dental professionals must make prudent decisions when selecting and donning PPE if they are to be effective in minimizing risks of exposure to infectious agents and accidents, which can threaten their health and safety as well as their patients. Most eye-related injuries can be prevented with proper education, adequate protective eyewear, and the correct handling of dental instruments and materials.

\section{References}

1) Douglas N. Dederich, Ronald D. Bushick, Lasers in dentistry: Separating science from Hype. JAm DentAssoc 2004; 135: 204 - 212.

2) Jolanta Szymanska, Work- related vision hazards in the dental office. Ann Agric Environ Med 2000; 7: 1-4.

3) Susan Lynn Tolle, Erin Kate Simmons, Maximizing protection: Dimensions of dental hygiene 2010; 8(3): 26, 28-31

4) Joyce Z. Downs, Neil D. Lutins, David W. Tybor: Common eye disorders encountered in the dental office: Dimensions of dental hygiene 2010; 8(3): 48-51

5) Peter A. Leggat, Ureporn Kedjarune, Derek R. Smith: Occupational health problems in modern dentistry: a review. Industrial health 2007; 45: 611-621

6) Caroline Sweeney. Laser safety in dentistry: General dentistry 2008; 56(7):653-659

\section{Congratulations}

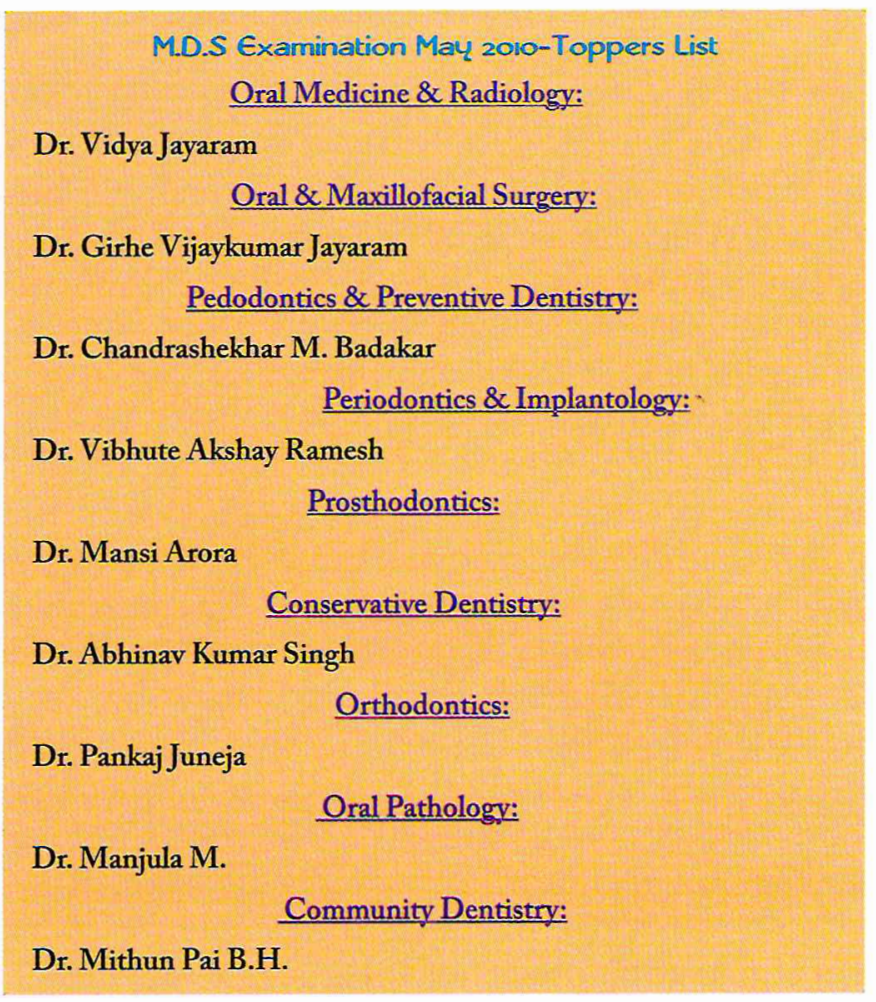

